\section{EDITORIAL}

Klin. Pädiat. 198 (1986) 135-136

(C) F. Enke Verlag Stuttgart

\title{
Die Hodgkin-Lymphom-Studien in der Pädiatrie - ein Beispiel für Effizienz und Kooperation*
}

F. Lampert

Universitäts-Kinderpoliklinik Gießen
Seit dem ersten Bericht vor 40 Jahren über schnellen Rückgang von Tumormassen durch die aus den Kampfgasen des 1. Weltkrieges entwickelten Stickstoff-Lostpräparate bei 27 Patienten mit Hodgkinscher Erkrankung (Goodman et al., 1946) ist die Lymphogranulomatose, der Morbus Hodgkin, das Paradebeispiel für die Wirksamkeit der Radiound Chemotherapie in der Onkologie. In dieser ersten Arbeit über Chemotherapie bei Morbus Hodgkin wurde bereits darauf hingewiesen, daß die Kombination von Bestrahlung und Chemotherapie wahrscheinlich jeder einzelnen Therapiemodalität überlegen sei, nur müsse noch das Optimale der Dosierungen, Kombinationen, zeitlichen Anwendung gefunden werden. Dieses Optimum zu erreichen, war das Ziel der Hodgkin-Lymphom-Studien der Deutschen Arbeitsgemeinschaft für Leukämieforschung und -behandlung im Kindesalter e. V.

Als in den 70er Jahren in der Bundesrepublik innerhalb der pädiatrischen Onkologie - viel früher als in der Erwachsenenonkologie - die ersten prospektiven, multizentrischen Therapiestudien sich etablierten, war die von G. Schellong, Münster, als Studienleiter inaugurierte M. Hodgkin-Studie die erste, die praktisch lückenlos alle Neuerkrankungen dieser Tumorart im Bundesgebiet erfaßte und ein Beispiel setzte für die Zusammenarbeit der Therapiemodalitäten, nämlich Chirurgie, Bestrahlung, Chemotherapie. Das Therapieziel bei Morbus Hodgkin, bei dem die potentielle Heilbarkeit schon seit Jahrzehnten bekannt war, lag auf der Hand: höchste Heilrate, geringste Therapiebelastung, und dies prospektiv überzeugend geprüft an einer Maximalzahl von Patienten unter kontrollierten Bedingungen. So selbstverständlich waren die Heilungen bei Morbus Hodgkin auch in den 70er Jahren beileibe noch nicht. Den Älteren unter uns werden noch die Zeiten erinnerlich sein, wo nicht wenige Kinder und Jugendliche an Morbus Hodgkin-Rezidiven verstarben, nach vorangegangenem mangelhaften Staging und entsprechend ungenügender Therapie.

Die DAL-HD 78-Studie (Breu et al., 1982) mit 170 Patienten des Kindes- und Jugendalters, rekrutiert aus $47 \mathrm{Be}-$ handlungsstätten, konnte zeigen, daß ein stabiles Heilniveau von über $90 \%$ bei allen Tumorausbreitungsstadien

* Herrn Prof. Dr. G. Schellong zum 60. Geburtstag gewidmet erreichbar war, und daß (durch Randomisierung geprüft), eine „Ausrottungsbestrahlung“ (36-40 Gy) nichtbefallener Lymphknotenregionen nicht notwendig war. Das wesentliche Erfolgsrezept dieser Studie war die der Bestrahlung vorangestellte Kombinationschemotherapie in Form des neu konzipierten OPPA-Blockes (Vincristin, Prednison, Procarbazin, Adriamycin), der bei allen, auch den niedrigen Ausbreitungsstadien in 2maliger Wiederholung angewandt wurde. Bei dieser prä-Bestrahlungs-Chemotherapie wurde bewußt das radiomimetisch wirkende und mit möglichen Spätschäden behaftete Cyclophosphamid weggelassen. Fester Bestandteil dieses Protokolls waren explorative Laparotomie und Splenektomie; ausgenommen davon waren nur Kinder unter 5 Jahren und einige mit Stadium IV. Dadurch war die Genauigkeit des prätherapeutischen „Staging“ gewährleistet. Als zukunftsträchtigste Frucht dieses ,Maximalvorgehens“ konnte nun in einer retrospektiven Analyse bei 154 Patienten (Schellong et al., 1982) gezeigt werden, daß ein Milzbefall bei der Bauchoperation zuverlässig durch 2 Befunde vorhergesagt werden konnte, nämlich durch ,,höckerige Veränderung der Milzoberfläche und/oder Vergrößerung der Lymphknoten am Milzhilus/ Pankreasschwanz". Die logische Folge war, daß in der Nachfolgestudie (DAL-HD 82) auf eine Splenektomie bei zwei Drittel der Patienten verzichtet werden konnte und die möglichen Postsplenektomiegefahren der Mehrzahl der Patienten zukünftig erspart werden konnten. Weiter wurden in der Nachfolgestudie vorsichtig bei Wahrung aller erreichten Heilchancen weitere ,Maximaltherapie"-Elemente abgebaut - so der völlige Verzicht auf Bestrahlung nichtbefallener Lymphknotenfelder, Reduzierung der Tumorfeldbestrahlungsdosis (35--25 Gy) und die Vorziehung nachgestellter Chemotherapie (COPP-Zyklen) bei den höheren Stadien in den Zeitraum vor die Bestrahlung, so daß überhaupt keine Chemotherapie nach der Bestrahlung mehr gegeben wurde. Der Erfolg bestätigte die Erwartungen: Die Auswertung der DAL-HD 82-Studie (Schellong et al., $1986 \mathrm{a}$; dieses Heft) nach $31 / 2$ Jahren bei über $200 \mathrm{~Pa}$ tienten unter $16 \mathrm{~J}$ ahren aus 53 Kliniken zeigte weiterhin ein ganz hohes Heilniveau (Wahrscheinlichkeit für ein erkrankungsfreies Überleben) mit $96 \%$ für die Gesamtgruppe (Stadium I/IIA: $99 \%$, IIB/IIIA: $96 \%$, IIIB/IV: $87 \%$ ). Nur bei $40 \%$ der Patienten wurde nach der neuen intraoperativen Entscheidungsstrategie die Milz entfernt. Ein Hodgkinbefall war bei $72 \%$ dieser Milzen nachweisbar. Bei den Patienten mit belassener Milz - immerhin zwei 
Drittel aller Patienten - wurde noch bei keinem einzigen ein Rezidiv beobachtet. Nach dieser erfolgreich praktizierten selektiven Splenektomie kann jetzt in der Nachfolgestudie DAL-HD 85 aufgrund von Daten der HD 82-Studie die selektive Laparotomie (bei auffälliger abdomineller Computertomographie/Sonographie und/oder LungenhilusLymphknotenbefall) praktiziert werden, so daß nur etwa die Hälfte aller Patienten mit Morbus Hodgkin bauchoperiert werden müssen (Schellong et al., 1986 b; dieses Heft).

Diese Studien der pädiatrischen Onkologie haben gezeigt, wie man folgerichtig und kontrolliert eine Maximaltherapie furr ein radiochemotherapeutisch sensibles Tumorleiden mit höchster Heilquote Zug um Zug doch so ,entschärfen“" kann, daß dem Patienten immer weniger Gefahren oder Folgeschäden entstehen können, - salus aegroti suprema lex -, auch in der Onkologie!

\section{Literatur}

1 Breu, H., G. Schellong, I. Grosch-Wörner, A. Jobke, H. Riehm, J. Ritter, J. Treuner, E.W. Schwarze, M. Wannenmacher: Abgestufte Chemotherapie und reduzierte Strahlendosis beim Morbus Hodgkin im Kindesalter - Ein Bericht über 170 Patienten der kooperativen Therapiestudie HD 78. Klin. Pädiat. 194 (1982) 233

2 Goodman, L.S., M.M. Wintrobe, W. Dameshek, M.J. Goodman, A. Gilman, M.T. McLennan: Nitrogen Mustard Therapy. Use of Methyl-Bis(Beta-Chloroethyl)amine Hydrochloride and Tris (Beta-Chloroethyl)amine Hydrochloride fo: Hodgkin's Disease, Lymphosarcoma, Leukemia and Certain Allied and Miscellaneous Disorders. J. Am. Med. Ass. 132 (1946) 126

3 Schellong, G., A.K. Waubke, H.J. Langermenn, H. Breu, B. Kuhne, H. Riehm, J. Ritter: Bedeutung klinischer und intraoperativer Befunde für die Voraussage eines Milzbefalls beim Morbus Hodgkin im Kindesalter: Eine retrospektive statistische Analyse bei 154 Patienten der Therapiestudie HD 78. Klin. Pädiat. 194 (1982) 242

4 Schellong, G., J. Brämswig, R. Ludwig, V. Gerein, A. Jobke, $H$. Jürgens, H. Kabisch, B. Stollmann, P. Weinel, H. Gadner, $E . W$. Schwarze, $M$. Wannenmacher: Kombinierte Behandlungstrategie bei über 200 Kindern mit Morbu: Hodgkin: Abgestufte Chemotherapie, involved-field-Bestrahlung mit erniedrigten Dosen und selektive Splenektomie. Ein Bericht der kooperativen Therapiestudie DAL-HD 82. Klin. Pädiat. 198 (1986) 137

5 Schellong, G., S. Lietzke, St. Strauch, B. Kühne, B. Schneider: Bedeutung sonographischer, computertom ographischer und klinischer Befunde für die Erkennung eines Abdominalbefalls beim Morbus Hodgkin im Kindesalter. Eine retrospektive statistische Analyse bei 145 Patienten der Therapiestudie DALHD 82. Klin. Pädiat. 198 (1986) 147

Prof. Dr. F. Lampert, Universitäts-Kinderpoliklinik, Feulgenstraße 12, D-6300 Gießen 